\title{
Distributed Spatio-Temporal Sampling of Diffusion Fields from Sparse Instantaneous Sources
}

\author{
Yue M. Lu and Martin Vetterli \\ School of Computer and Communication Sciences \\ Ecole Polytechnique Fédérale de Lausanne (EPFL) \\ Lausanne, CH-1015, Switzerland \\ Email: \{yue.lu, martin.vetterli\}@epfl.ch
}

\begin{abstract}
We study the spatio-temporal sampling of a diffusion field driven by $K$ unknown instantaneous source distributions. Exploiting the spatio-temporal correlation offered by the diffusion model, we show that it is possible to compensate for insufficient spatial sampling densities (i.e. sub-Nyquist sampling) by increasing the temporal sampling rate, as long as their product remains roughly a constant. Combining a distributed sparse sampling scheme and an adaptive feedback mechanism, the proposed sampling algorithm can accurately and efficiently estimate the unknown sources and reconstruct the field. The total number of samples to be transmitted through the network is roughly equal to the number of degrees of freedom of the field, plus some additional costs for in-network averaging.
\end{abstract}

\section{INTRODUCTION}

The diffusion process is widely used to model many important physical or biological phenomena. Some examples include temperature variations, air pollution dispersion, and photon transportation in tissues, to name a few. When the underlying medium is homogeneous and isotropic, the evolution of a diffusion field $f(\boldsymbol{x}, t)$, for $\boldsymbol{x} \in \mathbb{R}^{D}$, is governed by the heat equation [1]

$$
\frac{\partial f(\boldsymbol{x}, t)}{\partial t}=\mu \sum_{i=1}^{D} \frac{\partial^{2} f(\boldsymbol{x}, t)}{\partial x_{i}^{2}}+s(\boldsymbol{x}, t),
$$

where $\mu$ is the diffusion coefficient of the medium, and $s(\boldsymbol{x}, t)$ is the source of the field.

In this paper, we consider a particular class of source models

$$
s(\boldsymbol{x}, t)=\sum_{k=1}^{K} s_{k}(\boldsymbol{x}) \delta\left(t-t_{k}\right),
$$

which are sparse over time (i.e. containing $K$ Diracs), but nonparametric in space (i.e. consisting of $K$ smooth functions $\left.\left\{s_{k}(\boldsymbol{x})\right\}\right)$. In practice, such models can describe sudden events (e.g. explosions or accidental release of pollutants) that are the key targets of various environmental monitoring or security surveillance applications.

Figure 1 shows a diffusion field induced by three instantaneous sources. We observe such fields through a network of sensors, each taking samples over time. From these spatio-temporal samples, we want to accurately estimate the sources (including the spatial distributions $\left\{s_{k}(\boldsymbol{x})\right\}$ and time instants $\left.\left\{t_{k}\right\}\right)$ and faithfully reconstruct the field $f(\boldsymbol{x}, t)$.

Suppose that the spatial distributions $s_{k}(\boldsymbol{x})$ of the unknown sources are bandlimited, with a Nyquist density of $2 M+1$ samples per unit space. Then intuitively, with $K$ such distributions in the

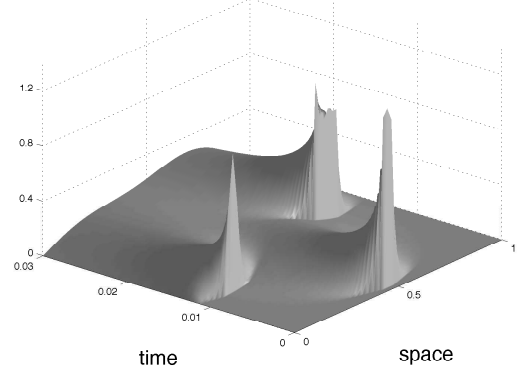

Fig. 1. A diffusion field induced by three instantaneous sources.

source model (2), we need to take at least $K(2 M+1)$ spatiotemporal samples to fully acquire the field. The question is, of course, how close this lower bound can be approached.

A more challenging issue is the trade-off between the spatial and temporal sampling densities. In practice, increasing the spatial sampling density is usually much more expensive than increasing the temporal sampling rate, since the former requires the physical presence of more sensors in the network, whereas the latter is, in theory, only constrained by the communication capacity and energy budget of the network. Given the different costs associated with spatial and temporal sampling, a natural question is whether one can compensate for insufficient spatial sampling densities by oversampling in time.

In this work, we address the above two questions by proposing an adaptive spatio-temporal sampling scheme, based on a network of $2 N$ sensors placed on nonuniform locations. The proposed scheme requires a total of $K(2 M+N)$ samples to be transmitted through the network, plus some additional costs for in-network averaging operations. Meanwhile, by exploiting the spatio-temporal correlation offered by the diffusion model, the number of sensors can be below the spatial Nyquist density of the field, i.e., $2 N<2 M+1$. With decreasing spatial densities, the temporal sampling rates need to increase accordingly so that their product remains roughly a constant (see Theorem 1 for a precise relationship).

The rest of the paper is organized as follows. Section II overviews some relevant concepts about the heat equation, which sets the ground for subsequent discussions. We then present the proposed spatio-temporal sampling scheme in three parts: In Section III, we study the reconstruction of the spatial distributions $\left\{s_{k}(\boldsymbol{x})\right\}$, assuming that the time instants $\left\{t_{k}\right\}$ are known. The problem of temporal parameter estimation is addressed in Section IV, based on a distributed sparse sampling algorithm. 
Finally, combining the two previous components, we summarize in Section $\mathrm{V}$ the main steps of the proposed adaptive spatiotemporal sampling scheme.

For simplicity of presentation, our following discussions only focus on the case of one spatial variable. Fortunately, the separable nature of the heat equation in (1) makes it convenient to extend our results to multiple spatial dimensions.

\section{The Structure of ThE Diffusion FiELD}

Let $f(x, t)$ be a diffusion field with one spatial variable $x \in$ $[0,1]$ and one temporal variable $t \in \mathbb{R}$. Consider the case where the field is initially all zero, and driven by $K$ instantaneous sources as in (2). The heat equation in (1) now becomes

$$
\frac{\partial f(x, t)}{\partial t}=\mu \frac{\partial^{2} f(x, t)}{\partial x^{2}}+\sum_{k=1}^{K} s_{k}(x) \delta\left(t-t_{k}\right) .
$$

Suppose that the unknown spatial distributions $\left\{s_{k}(x)\right\}$ are smooth, and can be well-represented by $(2 \mathrm{M}+1)$ Fourier bases

$$
s_{k}(x)=\sum_{m=-M}^{M} d_{m, k} e^{j 2 \pi m x}, \quad 1 \leq k \leq K,
$$

where $\left\{d_{m, k}\right\}_{m}$ are the Fourier coefficients of the $k$ th distribution $s_{k}(x)$. It follows that, at any time instant $t$, the field satisfying (3) can also be represented by $(2 M+1)$ Fourier bases [1]

$$
f(x, t)=\sum_{m=-M}^{M} c_{m}(t) e^{j 2 \pi m x} .
$$

Meanwhile, the time-varying Fourier coefficients $\left\{c_{m}(t)\right\}$ in (4) can be obtained as

$$
c_{m}(t)=\sum_{k=1}^{K} d_{m, k} e^{-\lambda_{m}\left(t-t_{k}\right)} U\left(t-t_{k}\right),
$$

where $\lambda_{m} \stackrel{\text { def }}{=} \mu(2 \pi m)^{2}$ and $U(t)$ represents the unit step function.

\section{Reconstructing the Spatial Distributions}

In this section, we focus on the problem of sampling and reconstructing the spatial distributions $\left\{s_{k}(x)\right\}$ of the sources, assuming that the time instants $\left\{t_{k}\right\}$ are known. The estimation of these temporal parameters is addressed in Section IV. Our discussions here extend some of our previous results in [2].

Let $\left\{c_{m, k} \stackrel{\text { def }}{=} c_{m}\left(t_{k}\right)\right\}_{m, k}$ denote the Fourier coefficients of the field at time $t_{k}$. We can verify from (5) that

$$
c_{m, 1}=d_{m, 1} \text { and } c_{m, k+1}=c_{m, k} e^{-\lambda_{m}\left(t_{k+1}-t_{k}\right)}+d_{m, k+1},
$$

for $1 \leq k<K$. The above relationship implies that the unknown source distributions $\left\{s_{k}(x)\right\}$ (with Fourier coefficients $\left\{d_{m, k}\right\}$ ) are uniquely determined by the Fourier coefficients $\left\{c_{m, k}\right\}$. Therefore, in what follows, we concentrate on reconstructing $\left\{c_{m, k}\right\}$.

Consider the following sampling setup, where we place $N$ sensors at spatial locations $0 \leq x_{1}<x_{2}<\ldots<x_{N} \leq 1$. Each sensor takes $L$ temporal measurements at time instants $\left\{\tau_{1}, \tau_{2}, \ldots, \tau_{L}\right\}$, where $t_{k} \leq \tau_{1}<\tau_{2}<\ldots<\tau_{L}<t_{k+1}$.

Betwen $t_{k}$ to $t_{k+1}$, the field undergoes pure diffusion with no additional source. Consequently, (4) and (5) can be simplified as

$$
f(x, t)=\sum_{m=-M}^{M} c_{m, k} e^{-\lambda_{m} t} e^{j 2 \pi m x}, \quad \text { for } t_{k} \leq t<t_{k+1} .
$$

Denote by $y_{n, \ell} \stackrel{\text { def }}{=} f\left(x_{n}, \tau_{\ell}\right)$ the $\ell$ th measurement taken at the $n$th sensor, and collect all these measurements into an $N$-by- $L$ matrix $\boldsymbol{Y} \stackrel{\text { def }}{=}\left[y_{n, \ell}\right]_{n, \ell}$. We can verify from (7) the following factorization

$$
Y=S C D,
$$

where $\boldsymbol{S}=\left[e^{j 2 \pi m x_{n}}\right]_{n,-M \leq m \leq M}$ is the "spatial sampling matrix", $\boldsymbol{D}=\left[e^{-\lambda_{m} \tau_{\ell}}\right]_{0 \leq m \leq M, \ell}$ is the "diffusion matrix", and $\boldsymbol{C}$ is the "Fourier coefficient matrix" defined as

$$
\boldsymbol{C}=\left(\begin{array}{cccc} 
& & c_{-(M-1), k} & \\
& . & & \\
c_{0, k} & & & \\
& \ddots & & \\
& & c_{M-1, k} & \\
& & & c_{M, k}
\end{array}\right) .
$$

There are two "extreme" cases where the Fourier coefficients $\left\{c_{m, k}\right\}$ can be straightforwardly obtained.

1) Satisfying the spatial Nyquist density: $N \geq 2 M+1$. In this case, the matrix $S$ always has a left inverse [3], and hence $\boldsymbol{S}^{\dagger} \boldsymbol{Y}=\boldsymbol{C D}$. The Fourier coefficients can then be read out by comparing the rows of $\boldsymbol{S}^{\dagger} \boldsymbol{Y}$ and $\boldsymbol{D}$. A clear drawback of this approach is that, when $M$ is large, we will need to use a large number of sensors in the network, significantly increasing the cost of the deployment.

2) Taking many temporal samples: $L \geq M+1$. In this case, the matrix $D$ is of full row rank (and hence has a right inverse) [4], owing to its generalized Vandermonde structure. It follows from (8) that, for $1 \leq m \leq M$,

$$
c_{m, k}(\boldsymbol{S})_{m}+c_{-m, k}(\boldsymbol{S})_{-m}=\left(\boldsymbol{Y} \boldsymbol{D}^{\dagger}\right)_{m},
$$

where $(\boldsymbol{S})_{m}$ and $(\boldsymbol{S})_{-m}$ represent the columns of $\boldsymbol{S}$ containing $\left\{e^{j 2 \pi m x_{n}}\right\}$ and $\left\{e^{-j 2 \pi m x_{n}}\right\}$, respectively. If these two column vectors are linearly independent, we can uniquely determine the Fourier coefficients from (9).

Proposition 1: A necessary condition for the Fourier coefficients $\left\{c_{m, k}\right\}_{m}$ to be uniquely determined from the spatiotemporal samples $\left\{y_{n, \ell}\right\}$ is that, for any $1 \leq m \leq M$, there exist two sensors $x_{n_{1}}, x_{n_{2}}$ in the network such that

$$
2 m\left(x_{n_{1}}-x_{n_{2}}\right) \notin \mathbb{Z} \text {. }
$$

The above condition is also sufficient when the number of temporal samples $L \geq M+1$.

Proof: (sketch) From (8), we see that for $\left\{c_{m, k}\right\}$ to be uniquely determined, the pair of column vectors $(\boldsymbol{S})_{m}$ and $(\boldsymbol{S})_{-m}$ must be linearly independent, for all $1 \leq m \leq M$. This requirement, together with the special complex exponential structure of the elements of $S$, leads to (10). The sufficiency of (10) when $L \geq M+1$ is due to (9).

Note that the condition (10) can be satisfied by using a network of only two sensors, placed at $x_{1}=0$ and $x_{2}=1 /(2 M+1)$, respectively. This implies that, in principle, we just need a total of two sensors to fully capture the field, provided that each sensor takes enough temporal samples. A caveat of this idealized scheme is that the condition number of the diffusion matrix $\boldsymbol{D}$ in (8) increases exponentially with $M$. Consequently, for large $M$, the reconstruction formula in (9) quickly becomes ill-conditioned.

In what follows, we propose a "middle ground" between the above two extremes. We want the number of sensors to be 
less than $2 M+1$ (i.e. spatial sub-Nyquist sampling), but the reconstruction should still be well-conditioned even for large $M$.

To that end, consider a set of sensor locations that is the union of two shifted uniform patterns

$$
\{n / N: 0 \leq n<N\} \cup\{n / N+s: 0 \leq n<N\},
$$

where $0<s<1 / N$. Note that when $s=1 /(2 N)$, the above scheme includes uniform sampling as a special case. Denote by

$$
a_{n}(t) \stackrel{\text { def }}{=} f(n / N, t), \quad b_{n}(t) \stackrel{\text { def }}{=} f(n / N+s, t)
$$

the field measurements at the two uniform patterns, respectively, and by

$$
\widehat{a}_{p}(t) \stackrel{\text { def }}{=} \sum_{n=0}^{N-1} a_{n}(t) e^{-j 2 \pi n p / N}, \quad \widehat{b}_{p}(t) \stackrel{\text { def }}{=} \sum_{n=0}^{N-1} b_{n}(t) e^{-j 2 \pi n p / N}
$$

their corresponding discrete Fourier transforms.

For every $p, 0 \leq p<N$, we can show that

$$
\begin{aligned}
& \widehat{a}_{p}(t)=N \sum_{u_{p} \leq i \leq v_{p}} c_{p+i N}(t), \quad \text { and } \\
& \widehat{b}_{p}(t)=N \sum_{u_{p} \leq i \leq v_{p}} c_{p+i N}(t) e^{j 2 \pi(p+i N) s}
\end{aligned}
$$

where $\left\{c_{m}(t)\right\}_{m}$ are the Fourier coefficients of the field defined in (4), $u_{p} \stackrel{\text { def }}{=} \min \{i \in \mathbb{Z}:|p+i N| \leq M\}$ and $v_{p} \stackrel{\text { def }}{=}$ $\max \{i \in \mathbb{Z}:|p+i N| \leq M\}$ are two integers. Due to space limitations, we omit the derivations of (14), which can be obtained by using the properties of the discrete Fourier transform and the definition of the field in (4).

For every $p$, we define a measurement matrix $\boldsymbol{Y}_{p}$ of size 2by- $L$, whose first row is $\left[a_{p}\left(\tau_{\ell}\right)\right]_{1 \leq \ell \leq L}$ and whose second row is $\left[b_{p}\left(\tau_{\ell}\right) e^{-j 2 \pi u_{p} s}\right]_{1 \leq \ell \leq L}$. From (14) and (7), we can easily verify the following factorization

$\boldsymbol{Y}_{p}=N\left(\begin{array}{ccccc}1 & 1 & 1 & 1 & 1 \\ 1 & e^{j 2 \pi N s} & e^{j 2 \pi N(2 s)} & \ldots & e^{j 2 \pi N\left(v_{p}-u_{p}\right) s}\end{array}\right) \boldsymbol{C}_{p} \boldsymbol{D}_{p}$,

where $\boldsymbol{C}_{p}=\operatorname{diag}\left\{c_{p+u_{p} N}, c_{p+\left(u_{p}+1\right) N}, \ldots, c_{p+v_{p} N}\right\}$ is a diagonal matrix of the Fourier coefficients, and $\boldsymbol{D}_{p}=$ $\left[e^{-\lambda_{p+i N} \tau_{\ell}}\right]_{u_{p} \leq i \leq v_{p}, \ell}$.

The factorization in (15) appears similar to that in (8). However, the main advantage here is that we have converted the original problem of dimension $2 M+1$ in (8) to $N$ independent problems in (15) (for different $p$ ), each of a much lower dimension $\left(v_{p}-u_{p}+\right.$ $1) \leq \frac{2 M}{N}+1$. Moreover, the formula in (15) can be parameterized by a single variable $s$. This structural simplicity allows us to obtain the following result.

Theorem 1: Suppose we use a network of $2 N$ sensors placed at locations according to (11). Each sensor takes $L$ temporal measurements between $t_{k}$ and $t_{k+1}$. If

$$
N(2 L-1) \geq 2 M,
$$

then there always exist suitable shifts $s$ in (11) such that any field of $2 M+1$ Fourier coefficients can be uniquely determined by the spatio-temporal measurements.

Proof: (sketch) Let $\boldsymbol{y}_{p}$ be the stacked vector from the columns of $\boldsymbol{Y}_{p}$, and $\boldsymbol{c}_{p}$ be the vector formed from the diagonal

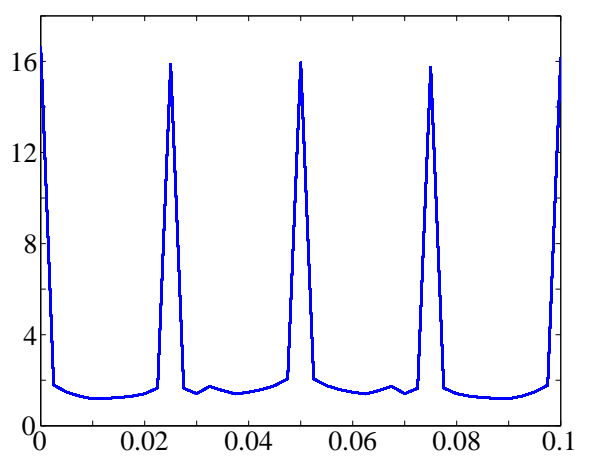

Fig. 2. The condition number (in natural log scale) of the reconstruction matrix $\boldsymbol{M}(\xi(s))$ for different values of the shift $s$.

elements of $\boldsymbol{C}_{p}$. Since (15) is linear with respect to $\boldsymbol{c}_{p}$, we can always rewrite (15) as

$$
\boldsymbol{y}_{p}=\boldsymbol{M}(\xi(s)) \boldsymbol{c}_{p}
$$

for some matrix $\boldsymbol{M}(\xi(s))$, where $\xi(s) \stackrel{\text { def }}{=} e^{j 2 \pi N s}$. On the one hand, we can show that $\operatorname{det}\left(\boldsymbol{M}^{*}(\xi) \boldsymbol{M}(\xi)\right)$ is a nonzero polynomial of $\xi$ of finite order, and hence allows only a finite number of zeros. For all the other values of $\xi$, the matrix $\boldsymbol{M}^{*}(\xi) \boldsymbol{M}(\xi)$ is nonsingular. On the other hand, the condition (16) guarantees that $\boldsymbol{M}(\xi)$ has no fewer rows than columns. Consequently, $\boldsymbol{M}^{*}(\xi) \boldsymbol{M}(\xi)$ being nonsingular implies that $\boldsymbol{M}(\xi)$ has full column rank and thus admits a left inverse.

Example 1: Consider a field that can be represented by 41 Fourier coefficients (i.e. $M=20$ ). We use a total of 20 sensors (i.e. $N=10$ ), placed on two uniform patterns shifted apart by a distance of $s$, with $0<s<0.1$. This setup corresponds to about two-times sub-Nyquist sampling in space. Each sensor takes $L=3$ samples over time, so that the condition (16) is satisfied. We plot in Figure 2 the condition number (in $\log$ scale) of the reconstruction matrix $\boldsymbol{M}(\xi(s))$ for different $s$. Confirming our argument in the proof of Theorem $1, M(\xi(s))$ is singular only for a finite number of $s$, and is otherwise invertible. The minimum condition number in this case is about 15 .

\section{Estimating the Temporal Parameters}

In this section, we propose a distributed sparse sampling scheme for estimating the unknown temporal parameters $\left\{t_{k}\right\}$ corresponding to the release time of the sources.

Recall from the previous section that the sensors are placed on two shifted uniform patterns, whose measurements are denoted by $\left\{a_{n}(t)\right\}_{n=1}^{N}$ and $\left\{b_{n}(t)\right\}_{n=1}^{N}$ as in (12). Let $g(t)$ represent the average value of all sensor measurements at time $t$, then

$$
g(t) \stackrel{\text { def }}{=} \frac{\sum_{n} a_{n}(t)+\sum_{n} b_{n}(t)}{2 N}=\frac{\widehat{a}_{0}(t)+\widehat{b}_{0}(t)}{2},
$$

where $\widehat{a}_{0}(t)$ and $\widehat{b}_{0}(t)$ are the DC components of the Fourier transforms in (13). Using the equalities in (14) and (5), and after some straightforward manipulations, we can compute $g(t)$ as

$g(t)=\sum_{k=1}^{K} d_{0, k} U\left(t-t_{k}\right)+\sum_{k=1}^{K} \sum_{i=1}^{\lfloor M / N\rfloor} \alpha_{k}^{i} e^{-\lambda_{i N}\left(t-t_{k}\right)} U\left(t-t_{k}\right)$,

where $\left\{\alpha_{k}^{i}\right\}$ are some constants. The second term on the righthand side of (17) are aliasing components, which appear when 
$N \leq M$. Taking the Fourier transform of (17),

$\widehat{g}(\omega)=\sum_{k=1}^{K}\left(d_{0, k}\left(\pi \delta(\omega)+\frac{1}{j \omega}\right)+\sum_{i=1}^{\lfloor M / N\rfloor} \frac{\alpha_{k}^{i}}{j \omega+\lambda_{i N}}\right) e^{-j \omega t_{k}}$.

It follows that

$$
(j \omega) \prod_{i=1}^{\lfloor M / N\rfloor}\left(j \omega+\lambda_{i N}\right) \widehat{g}(\omega)=\sum_{k=1}^{K} \sum_{i=0}^{\lfloor M / N\rfloor} \beta_{k}^{i}(j \omega)^{i} e^{-j \omega t_{k}},
$$

for some constants $\left\{\beta_{k}^{i}\right\}$.

Note that in the time domain, the right-hand side of (18) corresponds to a stream of differentiated Diracs

$$
h(t)=\sum_{k=1}^{K} \sum_{i=0}^{\lfloor M / N\rfloor} \beta_{k}^{i} \delta^{(i)}\left(t-t_{k}\right)
$$

The sampling and reconstruction of such signals have been previously studied in [5], [6], where it is shown that the parameters $\left\{t_{k}\right\}$ can be efficiently computed from the samples $\langle h(t), \varphi(t / T-n)\rangle$, where $\varphi(t)$ is some suitable sampling kernel of finite support (e.g. B-splines of sufficient order). In practice, the inner products can be obtained by applying a filter $\varphi(-t / T)$ to $h(t)$, followed by uniform sampling at every $T$ seconds.

To utilize the above result in our problem, we take the following samples $\langle g(t), \psi(t / T-n)\rangle$, where the kernel $\psi(t)$ in our case is obtained in the Fourier domain as

$$
\widehat{\psi}(-\omega T)=(j \omega) \prod_{i=1}^{\lfloor M / N\rfloor}\left(j \omega+\lambda_{i N}\right) \widehat{\varphi}(-\omega T) .
$$

It follows that $\langle g(t), \psi(t / T-n)\rangle=\langle h(t), \varphi(t / T-n)\rangle$.

An important issue is how to implement the above sampling scheme in a network setting with bandwidth constraints. Clearly, performing the filtering [by $\psi(t)$ ] and sampling all at a central processing unit (CPU) is infeasible, since this would require the CPU to know the spatial average $g(t)$ of the field at all time. A much more efficient scheme can be derived by noticing

$$
\begin{array}{r}
\langle g(t), \psi(t / T-n)\rangle=\left\langle\frac{\sum_{n} a_{n}(t)+\sum_{n} b_{n}(t)}{2 N}, \psi(t / T-n)\right\rangle \\
=\frac{\sum_{n}\left\langle a_{n}(t), \psi(t / T-n)\right\rangle+\sum_{n}\left\langle b_{n}(t), \psi(t / T-n)\right\rangle}{2 N} .
\end{array}
$$

In words, the order of the spatial averaging and linear sampling operations can be interchanged. Therefore, we can perform filtering and uniform sampling at each sensor locally in a distributed fashion. Only the low-rate samples taken at every $T$ seconds need to go through in-network averaging to the CPU, which then reconstructs the parameters $\left\{t_{k}\right\}$ from the samples.

\section{Adaptive Spatio-Temporal Sampling Scheme}

The CPU can estimate the parameters $\left\{t_{k}\right\}$ only after receiving enough samples of $\langle g(t), \psi(t / T-n)\rangle$. This delay poses a causality problem for the sampling scheme described in Section III, where we need the knowledge of $\left\{t_{k}\right\}$ in choosing the sampling instants (recall that for each $k$, every sensor needs to send $L$ samples taken between $t_{k}$ and $t_{k+1}$ ).

To solve this problem, we propose a feedback-based adaptive sampling scheme, summarized in the following procedure.

Procedure 1 (Adaptive spatio-temporal sampling): We use a network of $2 N$ sensors, placed at two shifted uniform patterns, to observe a diffusion field with spatial bandwidth $2 M+1$. Suppose that all $K$ sources of the field happen within a given observation window, i.e., $0 \leq t_{k}<W$ for some $W>0$. Meanwhile, consecutive time instants must be at least $\tau$ seconds apart.

1) Each sensor samples the field at every $\tau /(L+1)$ seconds, where $L$ satisfies (16). These samples are stored in a local buffer, which retains all such samples in the past $W$ seconds.

2) Each sensor sends out the samples $\langle f(x, t), \psi(t / T-n)\rangle$, where $T \leq W /(K\lfloor M / N\rfloor+K)$.

3) The network performs in-network averaging of these measurements and forward the results to a CPU.

4) From the sparse samples, the CPU runs a sparse reconstruction algorithm [5], [6] to estimate $\left\{t_{k}\right\}$.

5) The network returns the estimated $\left\{t_{k}\right\}$ to each sensor.

6) Each sensor pulls out $L$ samples taken between $t_{k}$ to $t_{k+1}$ from its buffer (this is always possible by the assumption that $\left.\left|t_{k+1}-t_{k}\right| \geq \tau\right)$, and forwards them to the CPU.

7) The CPU reconstructs the field at time $t_{k}$ by solving the linear equation in (15), and then obtains the spatial distribution of the source $s_{k}(x)$ according to (6).

The communication cost of the above scheme can be roughly estimated as

$$
K(2 N L) C_{1}+K(\lfloor M / N\rfloor+1) C_{2},
$$

where $C_{1}$ represents the average cost of sending one sample to the CPU, and $C_{2}$ denotes the cost of calculating the average value of the sensors via in-network averaging. Typically, $C_{2}<<2 N C_{1}$. Meanwhile, we can achieve an explicit trade-off between the number of sensors $2 N$ and the number of temporal samples $L$, as long as they satisfy the condition in (16).

\section{CONCLUSIONS}

We proposed a distributed spatio-temporal sampling algorithm for reconstructing diffusion fields induced by sparse instantaneous sources. The unknown temporal parameters of the sources are obtained by a distributed sparse sampling algorithm, and the spatial source distributions are reconstructed via linear inversion of spatio-temporal samples. For the latter, we exploit the spatiotemporal correlation offered by the diffusion model, and show that it is possible to achieve stable spatial sub-Nyquist sampling by temporal oversampling. The proposed adaptive sampling scheme can reconstruct the field efficiently, with the total number of samples transmitted through the network roughly equal to the number of degrees of freedom of the field, plus some additional costs for in-network averaging.

\section{REFERENCES}

[1] S. J. Farlow, Partial Differential Equations for Scientists and Engineers. New York, USA: Dover Publications, 1993.

[2] Y. M. Lu and M. Vetterli, "Spatial super-resolution of a diffusion field by temporal oversampling in sensor networks," in Proc. IEEE Int. Conf. Acoust., Speech, and Signal Proc., Taiwan, Apr. 2009.

[3] H. G. Feichtinger, K. Gröchenig, and T. Strohmer, "Efficient numerical methods in non-uniform sampling theory," Numer. Math, vol. 69, pp. 423440, 1995.

[4] D. Gilliam, Z. Li, and C. Martin, "Discrete observability of the heat equation on bounded domains," Int. J. Control, vol. 48, no. 2, pp. 755-780, 1988.

[5] M. Vetterli, P. Marziliano, and T. Blu, "Sampling signals with finite rate of innovation," IEEE Trans. Signal Process., vol. 50, no. 6, pp. 1417-1428, Jun. 2002.

[6] P. Dragotti, M. Vetterli, and T. Blu, "Sampling moments and reconstructing signals of finite rate of innovation: Shannon meets Strang-Fix," IEEE Trans. Signal Process., vol. 55, no. 5, pp. 1741-1757, May 2007. 